\title{
AC 2009-2191: DESIGN AND FABRICATION OF IMPACT (ACCELERATION) SENSORS AS CLASS PROJECTS IN A MEMS COURSE
}

\section{Mustafa Guvench, University of Southern Maine}

Dr. Mustafa G. Guvench received M.S. and Ph.D. degrees in Electrical Engineering and Applied Physics from Case Western Reserve University. He is currently a full professor of Electrical Engineering at the University of Southern Maine. Prior to joining U.S.M. he served on the faculties of the University of Pittsburgh and M.E.T.U., Ankara, Turkey. His research interests and publications span the field of microelectronics including I.C. design, MEMS and semiconductor technology and its application in sensor development, finite element and analytical modeling of semiconductor devices and sensors, and electronic instrumentation and measurement. 


\title{
Design and Fabrication of Impact (Acceleration) Sensors as Class Projects in a MEMS Course
}

\begin{abstract}
The paper describes use of SOI-MEMS fabricated Impact Sensors (Acceleration) as a real world design experience in an undergraduate senior level MEMS course. In addition to the standard lectures/reading/homeworks/tests routine of a typical coursework students were assigned to design, as individual class term projects, "Impact" or "Crash" Sensors using integrated circuit layout tools and standard Silicon MEMS technologies available and known as as "MUMPs" (Multi-User-MEMS-Processes). The Silicon-On-Insulator version of MUMPs which is named "SOI-MUMPs" was chosen. Successfully completed designs were combined to form a multi-project MEMS chip and sent out to be fabricated thanks to the funding received from NASA/MSGC. A test platform has been designed to create centrifugal g-forces up to $20 \mathrm{~g}$ 's. Currently, in the second offering of the course, students are testing these sensors to correlate the theory, analytical equations used for designs and the experimental results.
\end{abstract}

\section{Background and Description of the Work}

The paper describes use of SOI-MEMS fabricated Impact (Acceleration) Sensors as a real world design experience in a senior undergraduate level MEMS course.

This is a brand new technical elective in our Engineering programs, open to both electrical engineering and mechanical engineering students, on the emerging multidisciplinary field of Micro-Electro-Mechanical-Systems, better known as "MEMS". Topics covered include micromechanical structures, materials for MEMS and their thermal, electrical and mechanical properties, principles of microfabrication, micromechanics, electromechanical energy conversion and transduction. Basic electro-mechanical system blocks like beams, cantilevers, resonators, micro actuators are analyzed and their integration into the design of micro systems for applications in digital micro-mirror (DMM) projectors, acceleration and pressure sensors, RF and optical signal routers, micro pumps, micro motors and micro robots are discussed. Design tools for MEMS layout were demonstrated with the process steps and design rules of some standard MEMS fabrication available including "SOI-MUMPs". "MEMS-Pro" from SoftMEMS and "L-Edit" from Tanner were the preferred tools for their ease of use as well as the Windows operating system requirement familiar to all.

As a class design project students used these integrated circuit layout design tools demonstrated to design "Acceleration Sensors" as "Impact" sensors for application in vehicle air bag deployment. The designs had to employ the SOI (Silicon On Insulator) MEMS process, "SOI-MUMPs" available from MEMScAP. Students were given a set of constraints such as the maximum chip area available as well as the design rules specified by the 
company. Designs covered a range from about $3 \mathrm{~g}$ 's to $15 \mathrm{~g}$ 's. ("g" is the unit of acceleration measured equivalent to Earth's acceleration of gravity, i.e. $9.81 \mathrm{~m} / \mathrm{s}^{2}$.)

Thanks to the availability of funds from the instructor's NASA and MSGC grants, the student designs, after several feedback and corrections on them by the instructor to increase probability of success, were sent out for fabrication in the form of a multi-project chip which contains a total of nine such designs. In this way the cost of fabrication could be split among all nine. Figure 1 gives the layout design of the MEMS Impact Sensors Multi-Project chip containing all of the designs with initials of the students as well as the course number "ELE498" (Note the graffiti added on some designs by Red Sox fans in the class).

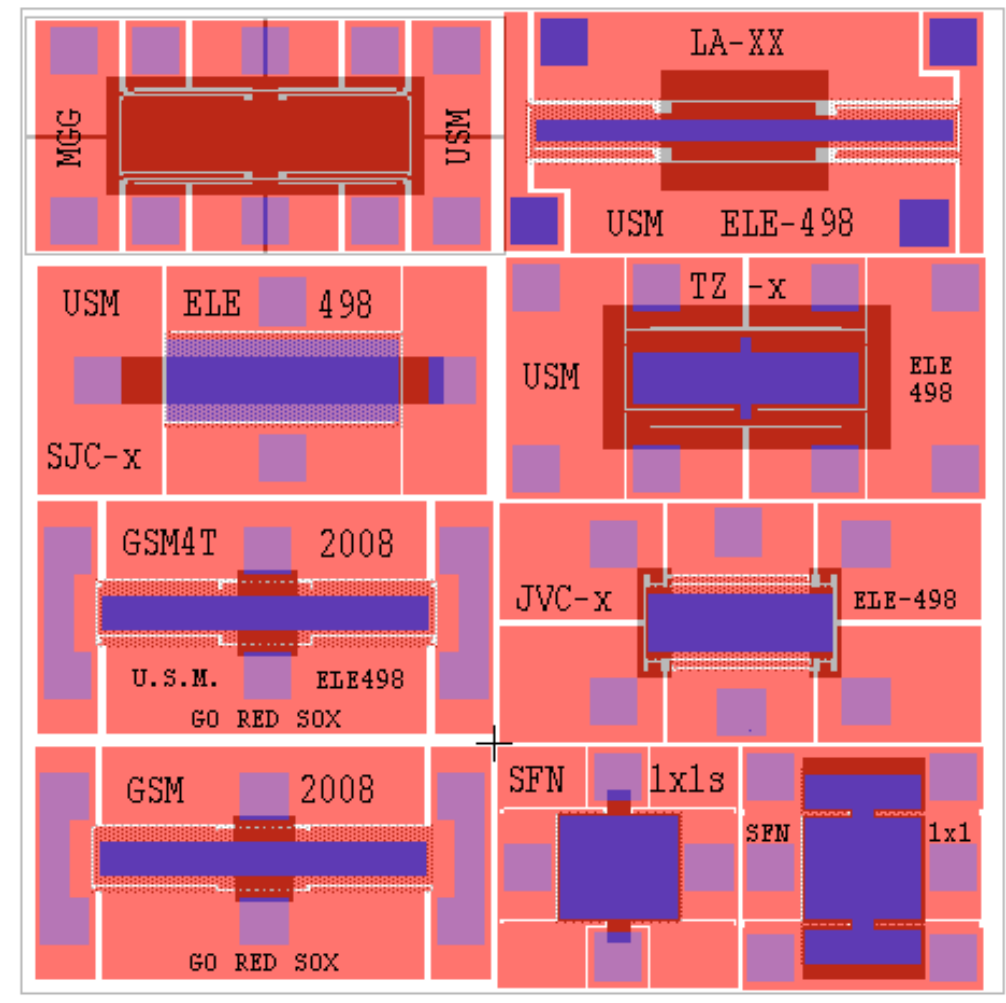

Figure 1 “ELE498 MEMS Impact Sensors” Multi-Project Chip Design Layout

All of these designs operate based on the fact that a shuttle mass hanging in air/vacuum tethered to a fixed frame, when subjected to acceleration forces, will be displaced with respect to the frame. The displacement will be limited by the balancing force of the thin tethers acting as springs. By forming, on the MEMS structure, electrical contacts whose gaps are calculated to make contact at the set threshold value of acceleration (or the crash point for the deployment of the air bag in a vehicle) impact sensors are created. The threshold impact acceleration (or the point) is, then, given by,

$$
a_{\text {impact }}=\left(N_{\text {tethers }} \cdot K_{\text {tethers }} \cdot d_{\text {gap }}\right) / M_{\text {shuttle }}
$$

, where $\mathrm{N}_{\text {tethers }}=4$ is the number of tethers, $\mathrm{K}_{\text {tethers }}$ is the spring constant of each tether, $\mathbf{M}_{\text {shuttle }}$ is the mass of the shuttle and $\mathrm{d}_{\text {gap }}$ is the contact gap. Obviously, design of such an 
apparently simple device becomes challenging due to the microscopic size of the shuttle, material properties of Silicon, integrated circuit layout design rules imposed by the MEMS fabrication process and others such as the real estate area available for each design on the multi-project chip. A total of 9 designs were fitted into a $4.5 \mathrm{~mm}$ x $4.5 \mathrm{~mm}$ multi-project die (shown in Figure 1).

Figure 2 gives a 3-D picture of one of the student designs. Both top view (on the left) and bottom view (on the right) are shown. Since the mass of such a tiny shuttle (about $200 \mu \mathrm{m} \mathrm{x}$ $1400 \mu \mathrm{m} \times 10 \mu \mathrm{m}$ size carved in Silicon) is about 7 nano-grams, the tethers have to have a very small spring coefficient, therefore they have to be very thin $(2 \mu \mathrm{m}$, the smallest feature size allowed in SOI-MUMPs process) and the contact gaps have to be minimal $(2-3 \mu \mathrm{m})$. That is why in Figure 2 the tethers and the gaps are hard to see. In those $3 \mathrm{D}$ drawings red represents the $10 \mu \mathrm{m}$ thick Silicon which is resting on a $400 \mu \mathrm{m}$ thick Silicon substrate coated with a $1 \mu \mathrm{m}$ insulating oxide layer, and the yellow areas are the Gold coatings to reduce the contact resistance of the switch and to form wire bonding pads.

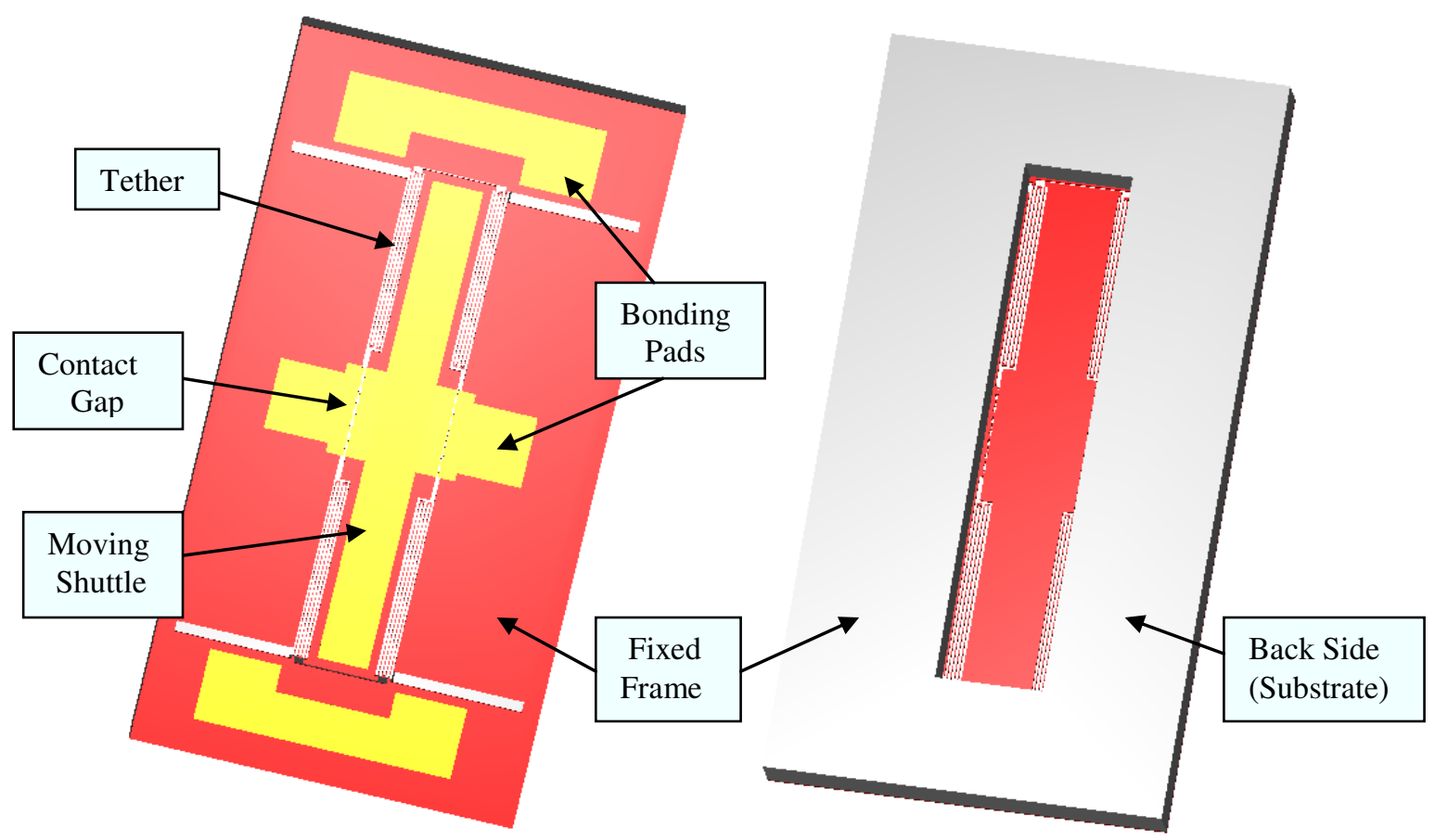

Figure 2. A Sample of Student Designs (GSM) - Top and Bottom Views

Due to the long time needed for the fabrication of the chip testing is left to the next class. Currently wire bonding of the chips and building of electronic test circuits as well as building of a platform for the creation of acceleration forces up to $20 \mathrm{~g}$ 's are being completed for the final testing and verification of the MEMS Impact Sensors designed. Figure 3 shows the photo of the acceleration test platform designed. It consists from a $1 \mathrm{~m}$ long metal bar 
attached to a shaft at its center. The shaft is attached to a speed reducing gear and is driven by a DC motor. It can spin up to $180 \mathrm{rpm}$ at maximum power, creating close to a maximum centrifugal force of about $20 \mathrm{~g}$ 's on a test chip plugged in to the circuit boards fixed inside the metal boxes. An Analog Devices AD321XL acceleration sensor which is placed inside the test chamber along with the MEMS chip under test, acts as a reference to measure and monitor the acceleration force experienced by the MEMS chip as the speed of rotation is gradually increased to cover an acceleration test range of 0 to 20 g's.

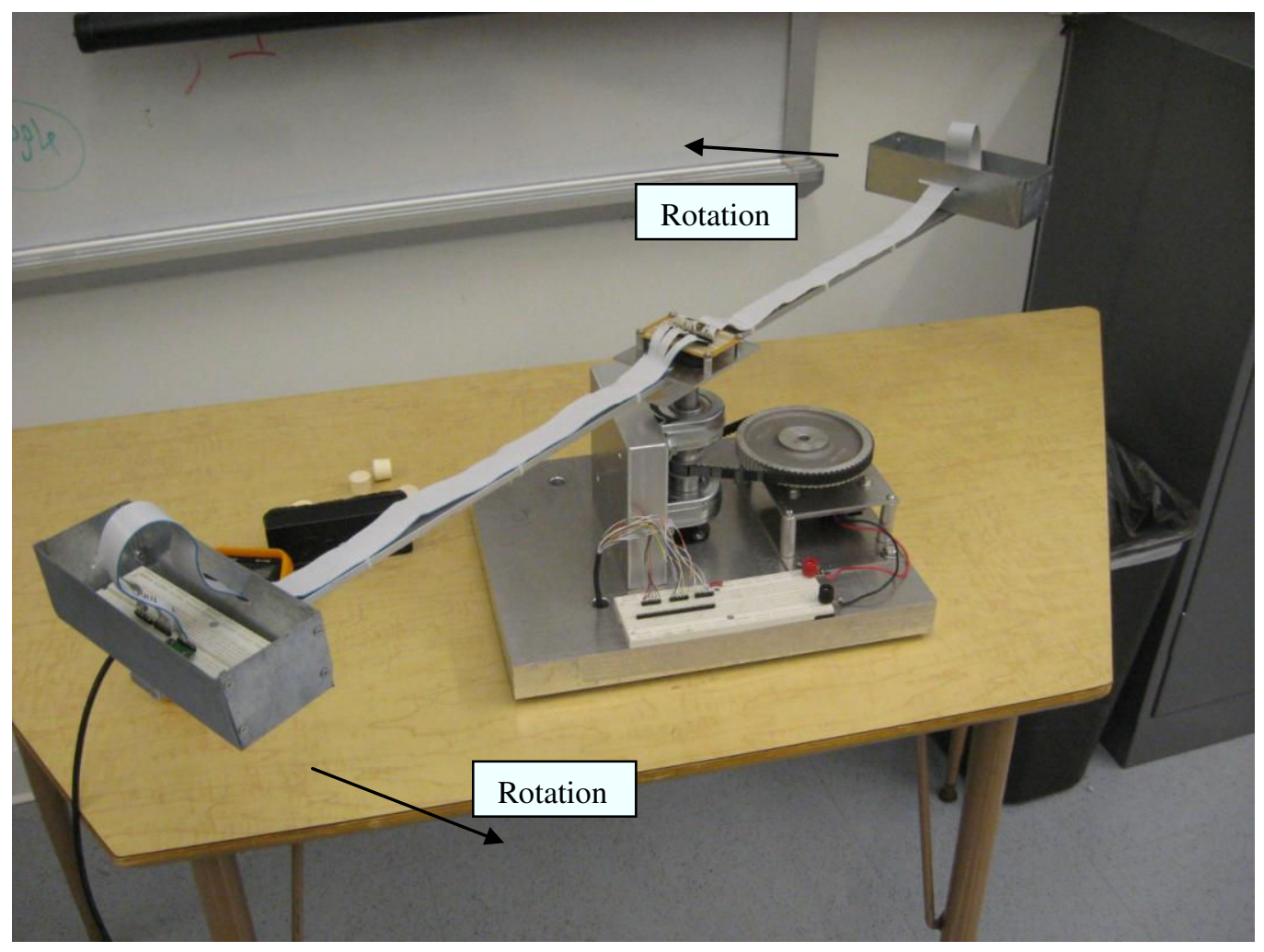

Figure 3. Acceleration Sensor Test Platform

Figure 4 gives a comparison of test results in comparison with design calculations and COMSOL simulations. These simulations were done by a new group of students during the second offering of the course by using the layout information and the dimensions of the structures made available to them. 


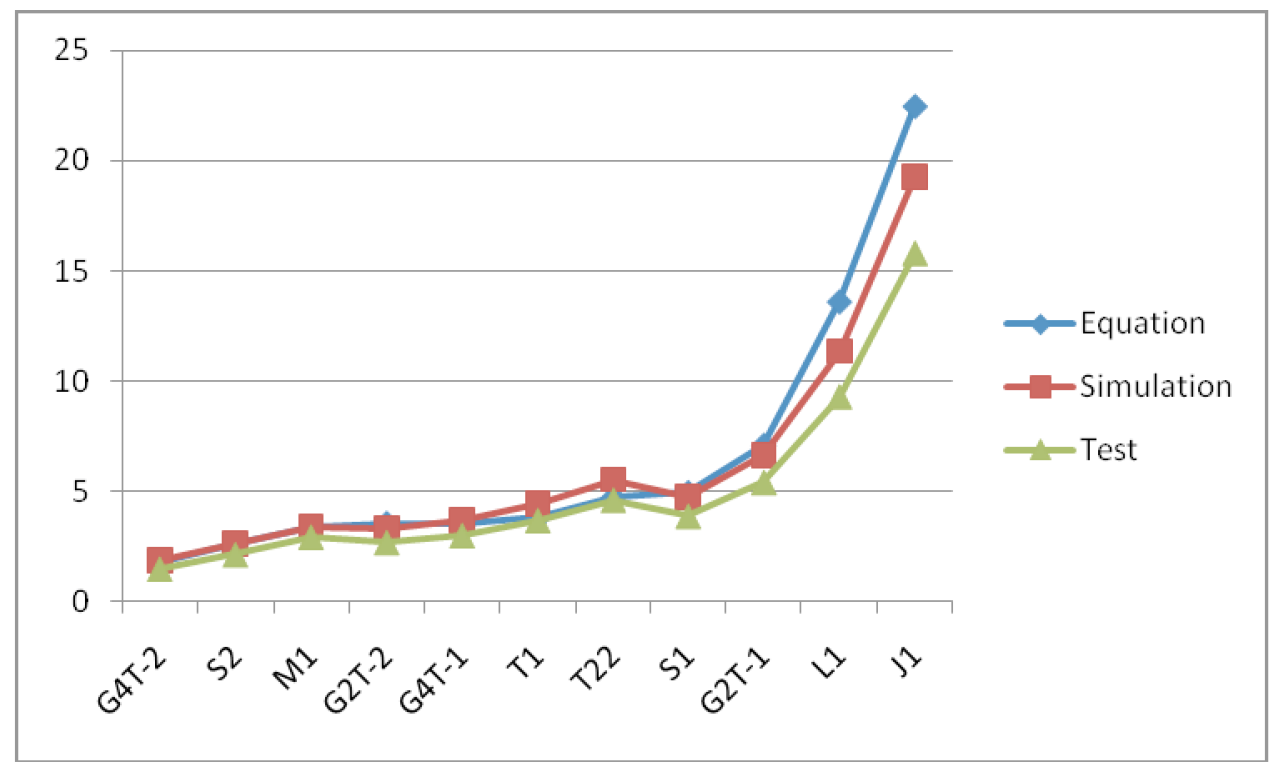

Figure 4. Acceleration values in g's for various designs

\section{Results and Conclusions}

Preliminary results on the acceleration tests follow the general trend of the lump model calculations using Equation (1) and the spring model and the material parameters taken from Liu [2]. However, there is up to $20 \%$ difference observed between the model and the experiment. The spring model is "fixed-guided beam under point loading at the free end" which predicts individual tether spring constant as $\mathrm{K}=12 \mathrm{E} . \mathrm{I} / \mathrm{l}^{3}$, where $\mathrm{E}$ is the young's modulus, $\mathrm{l}$ is the length of the beam and $\mathrm{I}$ is the moment of inertia of the beam's cross section. Young's modulus was taken as $160 \mathrm{GPa}$. In all designs, in order to achieve small spring coefficients for high sensitivity, long and folded beams have been used. In the design calculations which are based on the lumped model it was assumed the effects of joints and end points were negligible, and the spring constant is simply divided by the number of the folded sections. Assuming isotropic material properties (constant Young's modulus) and perfectly rectangular and uniform cross section for the beams, the spring constant can be written as,

$$
\mathrm{K}_{\text {tether }}=\mathrm{E} \cdot \mathrm{t}(\mathrm{w} / \mathrm{l})^{3} / \mathbf{n}_{\text {folding }}
$$

where $t$ is the thickness, $w$ is the width, 1 is the length of each section of the beam, and $n_{\text {folding }}$ is the number of times the beam is folded. When combined with equation (1), the threshold impact acceleration of equation (1) can be written as,

$$
a_{\text {impact }}=N_{\text {tethers }}\left(\text { E. } t(w / l)^{3} / n_{\text {folding }}\right) \cdot d_{\text {gap }} / M_{\text {shuttle }}
$$

This is the model equation used in our design calculations. It is to be noted that, the lithography and etching control and the uniformity limit of 0.1 um of process, with typical $w$ and $d_{\text {gap }}$ values used in our designs (about $2 \mu$ minimum allowed values for width and separation) amounts to $5 \%$ uncertainty in each. Total contribution of these two on the impact acceleration number of equation (3) can be as much as $(1.05)^{4}-1=21 \%$ uncertainty. This alone explains the deviation of the actual results from our calculated values. 
In conclusion, inclusion of this full cycle design experience, the testing of the fabricated chips and the finite element modeling work initiated with the second generation of the students in our MEMS class gave our students an extraordinary engineering experience, made the course popular and has almost doubled the enrollment in class, and attracted students from mechanical engineering seniors (about $25 \%$ of the class).

\section{Acknowledgements}

This project would not have been possible without the research and educational grants received from NASA and MSGC. It was these grants that paid for the fabrication of the MEMS chips. The author thanks his students for their enthusiastic participation in the projects, particularly Greg Mitchell who also helped to construct the acceleration sensor testing platform.

\section{REFERENCES}

[1] T.H. Hsu, " MEMS \& Microsystems: Design, Manufacture" McGraw Hill 2001 and JW 2008

[2] C. Liu, "Foundations of MEMS" Prentice Hall 2005.

[3] R.C. Jaeger, "Intr. to Microelectronic Fabrication, $2^{\text {nd }}$ ed.," Addison Wesley 2002

[4] J.P. Uyemura, "Physical Design of CMOS Integrated Circuits Using L-EDIT", PWS 1995

[6] MEMS-Pro ${ }^{\mathrm{TM}}$ is a product of SoftMEMS, http://www.softmems.com

[7] $\mathrm{L}-E d i t^{\mathrm{TM}}$ is a product of Tanner Research, http://www.tanner.com 